\title{
New Insight into the Treatment of Advanced Differentiated Thyroid Cancer
}

\author{
Arash Safavi, ${ }^{1}$ Aparna Vijayasekaran, ${ }^{1}$ and Marlon A. Guerrero', ${ }^{1,2}$ \\ ${ }^{1}$ Department of Surgery, University of Arizona, Tucson, AZ 85724-5131, USA \\ ${ }^{2}$ The University of Arizona Cancer Center, Tucson, AZ 85719-1454, USA \\ Correspondence should be addressed to Arash Safavi, asafavi@email.arizona.edu
}

Received 3 November 2012; Accepted 12 December 2012

Academic Editor: Maria Grazia Chiofalo

Copyright ( $) 2012$ Arash Safavi et al. This is an open access article distributed under the Creative Commons Attribution License, which permits unrestricted use, distribution, and reproduction in any medium, provided the original work is properly cited.

\begin{abstract}
The vast majority of patients with differentiated thyroid cancer (DTC) are treated successfully with surgery and radioactive iodine ablation, yet the treatment of advanced cases is frustrating and largely ineffective. Systemic treatment with conventional cytotoxic chemotherapy is basically ineffective in most patients with advanced DTC. However, a better understanding of the genetics and biologic basis of thyroid cancers has generated opportunities for innovative therapeutic modalities, resulting in several clinical trials. We aim to delineate the latest knowledge regarding the biologic characteristics of DTC and to describe the available data related to novel targeted therapies that have demonstrated clinical effectiveness.
\end{abstract}

\section{Introduction}

Thyroid cancer, the most common endocrine malignancy, constitutes about $1 \%$ of all malignancies. Differentiated thyroid cancer (DTC) includes papillary (PTC), follicular (FTC), and Hürthle cell (HCC) carcinoma, accounting for more than $90 \%$ of all thyroid cancers $[1,2]$.

The incidence of thyroid cancer has been steadily increasing over the last 20 years. In the United States alone, an estimated 56,460 new cases will be diagnosed in 2012; of those cases, $75 \%$ will occur in women [3]. In spite of the rising incidence, the death rate has remained relatively constant; in 2012, the American Cancer Society expects a total of about 1,780 deaths [3]. Across the world, the annual incidence of DTC varies, affecting an estimated 1.2 to 2.6 men per 100,000 individuals and an estimated 2.0 to 3.8 women per 100,000 individuals.

Most cases of DTC are slowly progressive; when identified at an early stage, patients can frequently be cured with adequate surgical care and radioactive iodine 131-I (RAI) ablation therapy. But advanced DTC-defined as thyroid cancer that has become metastatic, is inoperable, or is refractory to RAI therapy—is associated with a poor prognosis [4].
Cytotoxic chemotherapy and radiotherapy are essentially ineffective for most patients with advanced DTC. Over the last decade, a better understanding of the genetics and biologic basis of thyroid cancers has generated opportunities for innovative therapeutic modalities $[5,6]$. In this paper, we aim to delineate the latest knowledge regarding the biologic characteristics of DTC and to describe the available data related to novel targeted therapies that have demonstrated clinical effectiveness.

\section{Materials and Methods}

Using the MEDLINE, Embase, and Cochrane databases, we carried out a literature search. We used the specific terms "metastatic thyroid cancer," or "advanced thyroid cancer," and "targeted therapies," as well as the name of each individual agent. For review and possible inclusion in our study, we selected only relevant articles (describing either clinical experience or trials) published in English from January 2000 through September 2012. In addition, we checked ClinicalTrials.gov for information on registered clinical trials; we searched for any results of trials, using the keywords "thyroid neoplasm" and "randomized," and then incorporated results we found into our study. 


\section{Results}

\subsection{Potential Molecular Targets}

3.1.1. Papillary Thyroid Cancer (PTC). Mutations of the $\mathrm{B}$ isoform of RAF kinase (BRAF mutations) have been reported in about $40 \%$ to $70 \%$ of PTC tumors [24, 25]. The $B R A F^{T 1799 A}$ mutation is the most common genetic change in PTC, which leads to a V600E amino acid substitution and then to constitutive activation of the BRAF kinase, and hence upregulation of downstream pathways [26].

RET/PTC mutations are also well known as one of the most common mutations in PTC. At least 15 types of RET/ PTC rearrangements have been described [27, 28]. Some studies have underscored that RET rearrangements are associated with the lack of transformation of PTC into poorly differentiated or anaplastic carcinoma $[29,30]$.

$B R A F^{T 1799 A}$ (V600E) mutations are exclusive to PTC: they are not found in any other forms of DTC or in follicular (FTC), Hürthle cell (HCC), or medullary thyroid cancer [26]. No overlap seems to exist between RET/PTC, BRAF, or RAS mutations in PTC [31]. On the other hand, BRAF ${ }^{T 1799 A}$ (V600E) is a definitive marker of malignancy, as it has never been found in benign nodules while RET/PTC has been found in nonmalignant thyroid nodules. The biological and clinical significance of RET/PTC in benign thyroid lesions is still controversial [32, 33].

NTRK1 rearrangements are considerably less frequent in PTC than are RET rearrangements. The rate of NTRK1 rearrangements was about $3 \%$ in cases of post-Chernobyl PTC [34]. All these mutations potentially lead to upregulation of the RAS/BRAF/mitogen-activated protein kinase (MAPK)/extracellular signal-related kinase (ERK) kinase (MEK)/ERK or MAPK pathway that is involved in thyroid cancer.

3.1.2. Follicular Thyroid Cancer (FTC). In a comparative study of RAS mutations and PPAR $\gamma$ rearrangements, $49 \%$ of conventional FTC tumors had RAS mutations, 35\% had PAX8-PPAR $\gamma$ rearrangements, and only $3 \%$ had both abnormalities; $12 \%$ were negative for both [35]. In some studies, PAX8-PPAR 1 rearrangements have been reported in about $53 \%$ of conventional FTC tumors [36-38]. About 20\% to $30 \%$ of FTC tumors in another study had the PTEN mutation [37]. The PI3K pathway may also be activated in a few cases of papillary and follicular cancer [38, 39]. The PI3KAkt pathway is an alternate pathway to the MAPK pathway. In aggressive DTC and anaplastic thyroid cancer mutations that upregulate, both pathways are common [40].

In a pooled analysis of 229 cases of $R A S$ mutations using 39 previous publications, Vasko et al. compared the findings with data from their own cohort of 80 patients. Their analysis showed that mutations of codon 61 of N-RAS (N2) were significantly more frequent in FTC (19\%) than in PTC (5\%) and significantly more common in malignant $(25 \%)$ than in benign (14\%) tumors. H-RAS mutations in codons 12/13 (H1) were found in 2-3\% of all types of tumors, but H-RAS mutations in codon $61(\mathrm{H} 2)$ were observed in only $1.4 \%$, and almost all of them were malignant. K-RAS mutations in exon 1 were found more often in PTC than FTC (2.7\% versus $1.6 \%)[41]$.

Molecular targeted therapies are being developed to target the aforementioned specific mutations and pathways. Tyrosine kinase inhibitors are especially critical because of their important role in the modulation of growth factor: they signal and interrupt the pathologically activated pathways and thereby affect tumor growth. Each tyrosine kinase compound may or may not be exclusive to a single cascade. However, several interconnections exist between various signaling pathways, so a therapeutic spectrum that is too focused may not be optimal $[42,43]$.

In addition to targeting specific mutations and pathways, molecular-targeted therapies are being developed to target the blood vessels of the tumor, either by a drug-induced obliteration of already-established vessels or by a prompting of the signaling pathways to commence the growth of new blood vessels. In experimental models, manipulation of vascular functions regulated by vascular endothelial growth factor (VEGF) has blocked the growth of DTC [44]. Numerous VEGF subtypes and VEGF receptors-including VEGFR1 (FLT1) and VEGFR-2 (KDR) - as well as receptors for fibroblast growth factor (FGF) and platelet-derived growth factor (PDGF) are frequently overexpressed in the vascular endothelium of thyroid cancers and also trigger the MAP kinase signaling pathway [45]. Therefore, therapeutically blocking the function of angiogenesis factors might be an important concurrent approach that will improve the outcome of patients with DTC.

\subsection{Therapeutic Compounds}

3.2.1. Tyrosine Kinase Inhibitors. Recently completed phase 1 and 2 clinical trials suggest that tyrosine kinase inhibitors confer a clinical benefit in patients with DTC. However, not all of these agents have achieved U.S. Food and Drug Administration (FDA) regulatory approval for the treatment of patients with advanced DTC; few are FDA-approved for the treatment of other carcinomas. The mechanism and outcome of these agents are summarized in Table 1.

\subsection{Clinical Trials}

Motesanib (125 mg/day). The First large, international trial of a tyrosine kinase inhibitor in patients with progressive DTC was a phase 2 study of motesanib. Of 93 enrolled patients, $14 \%$ had a partial response; stable disease was achieved in $67 \%$ of the patients and was maintained for 24 weeks or longer in $35 \%$. The median progression-free survival (PFS) time was 40 weeks [7]. Interestingly, tumors with BRAF mutations responded better than BRAF-negative tumors, despite the absence of BRAF inhibition by motesanib, suggesting that these BRAF-positive tumors might be more dependent on other signaling pathways. The most common treatment-related adverse events were diarrhea, hypertension, fatigue, and weight loss.

Axitinib (5 mg twice daily). In a multicenter phase 2 study of 45 DTC patients, axitinib induced a partial response in 14 


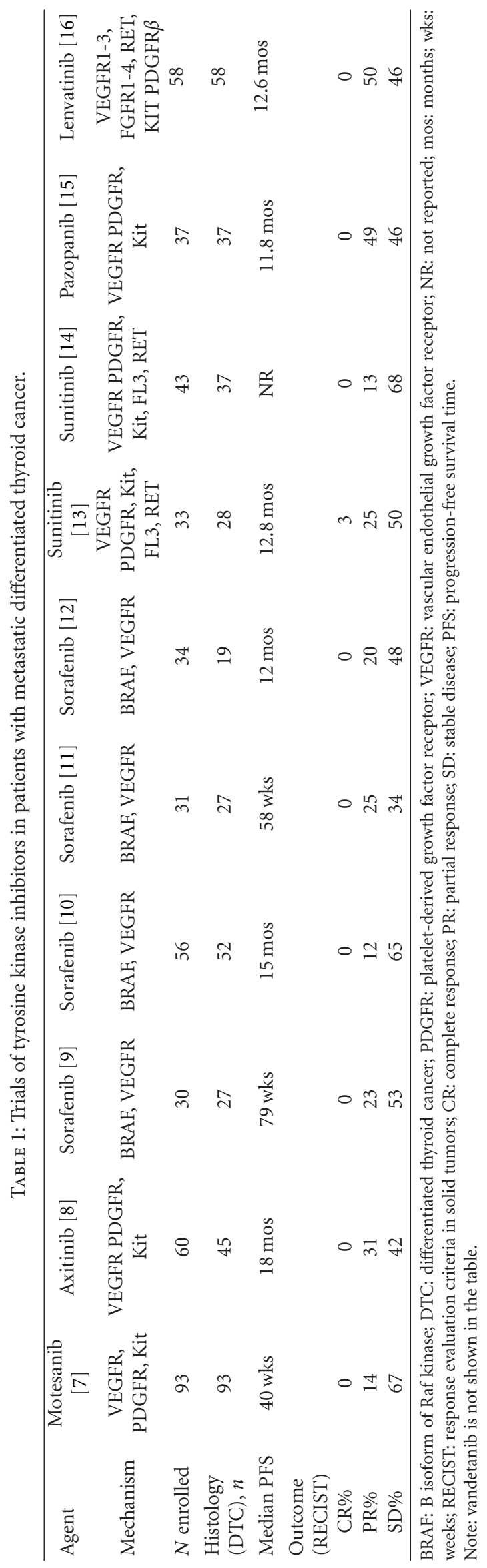


(31\%); stable disease was achieved for more than 16 weeks in another 19 (42\%) patients. The median progression-free survival for all histologic types of thyroid cancer was 18 months. Reported adverse drug effects were hypertension, proteinuria, fatigue, diarrhea, weight loss, and headache [8].

Sorafenib (400mg twice daily). Four phase 2 trials have reported on the effect of sorafenib in DTC patients. One trial included 30 DTC patients: 7 had a partial response and another 16 had stable disease [9]. The second trial, a larger one sponsored by the National Cancer Institute, included 52 DTC patients: $12 \%$ had a partial response and another $65 \%$ had stable disease [10]. The third trial included 31 DTC patients: 8 had a partial response and another 11 had stable disease for more than 24 weeks [11]. The fourth trial included 19 DTC patients: 20\% had a partial response [12]. The most common adverse drug effects were hypertension, fatigue, weight loss, palmar-plantar erythema, colon perforation, other skin toxicities, diarrhea, alopecia, and arthralgia [9-12].

With early data showing sorafenib to have promise in DTC patients and with sorafenib being commercially available (in the United States, for patients with advanced renal cell carcinoma or unresectable hepatocellular carcinoma), many clinicians began using it in an off-label manner [12]. In a retrospective analysis of sorafenib in 7 Spanish referral centers, DTC patients who were not suitable for curative surgery, for RAI therapy, or for radiotherapy were treated with sorafenib (400 mg twice daily); 3 of 6 DTC patients had a partial response (median follow-up, 11.5 months) [46]. Marotta et al. assessed the impact of off-label sorafenib in a cohort of 17 patients with progressive DTC refractory to radioactiveiodine. Clinical benefit was obtained in $71 \%$ of subjects $(30 \%$ partial response and $41 \%$ stable disease; median followup was 15.5 months). Sorafenib was mostly well tolerated, but a high incidence of fatal events was reported (3 patients died from severe bleeding events and 2 from cardiac arrest) [13]. Now in progress is a phase 3 trial comparing the effect of sorafenib versus placebo on progression-free survival in patients with progressive metastatic DTC not responsive to RAI therapy (NCT00984282).

Sunitinib (37.5 mg daily). In a phase 2 trial by Carr et al. of sunitinib in 28 DTC patients, 1 (3\%) had a complete response; 7 (25\%), a partial response; and another $14(50 \%)$, stable disease [14]. In a second phase 2 trial by Cohen et al. of sunitinib (50 mg/day, 4 weeks on and 2 weeks off) in 31 DTC patients with progressive metastases, 13\% had a partial response; 68\%, stable disease [15]. Reported adverse drug effects were fatigue, neutropenia, leukopenia, diarrhea, gastrointestinal (GI) bleeding, supraventricular tachycardia, anemia, dehydration, mucositis, odynophagia, thrombocytopenia, laryngeal edema, hypocalcemia, peripheral neuropathy, and infection $[14,15]$. Sunitinib is currently FDAapproved and available in the United States to treat patients with renal cell carcinoma.

Pazopanib ( $800 \mathrm{mg}$ daily). In a Mayo Clinic phase 2 trial of pazopanib in 37 DTC patients, $49 \%$ had a partial response.
The likelihood of a response lasting longer than 1 year was calculated to be $66 \%$; the PFS time at 1 year, $47 \%$ [16]. Adverse drug effects were elevated liver enzymes, lower GI bleeding, hypertension, weight loss, colonic perforation, diarrhea, oral mucositis, hypoalbuminemia, hypokalemia, abdominal pain, chest pain, headache, cough, and raised creatinine levels. Pazopanib is currently FDA-approved and available in the United States to treat patients with advanced renal cell carcinoma.

Lenvatinib (E7080) (24 mg daily). In a phase 2 trial of lenvatinib in 58 patients, $50 \%$ had a partial response $(54 \%$ for "naïve" or newly treated patients, $41 \%$ for pretreated patients); 46\%, stable disease. The median PFS time was 13.3 months. The dose was reduced in 39\% of patients and withdrawn in 29\% [47]. Based on these results, a phase 3 trial will soon be initiated to compare the effect of lenvatinib versus placebo on PFS time in patients with progressive refractory DTC (NCT01321554).

Vandetanib (300 mg/day). Vandetanib is the first and only targeted drug to show evidence of efficacy in a randomized trial. In the first randomized phase 2 study of a kinase inhibitor in DTC patients, 145 patients from 16 centers across Europe were recruited for placebo versus vandetanib arms [48]. The vandetanib patients had a longer median PFS time than the placebo patients (hazard ratio (HR), 0.63; $60 \%$ confidence interval (CI), 0.54 to 0.74 ; one-sided $P=$ 0.008). For the vandetanib patients, the median PFS time was 11.1 months (95\% CI, 7.7 to 14.0$)$; for the placebo patients, 5.9 months ( $95 \%$ CI, 4.0 to 8.9). The most common adverse events were QTc prolongation, diarrhea, asthenia, and fatigue.

Sorafenib ((400 mg each morning, $200 \mathrm{mg}$ each evening) and Tipifarnib (100 mg twice daily)). In a phase 1 trial, 35 DTC patients were treated for 21 days in each 28-day cycle (with 7 days off): of 22 DTC patients, $1(4.5 \%)$ had a partial response and $8(36 \%)$ had stable disease for at least 6 months. The median PFS time for all 35 patients was 18 months [49].

Cabozantinib (XL184) (140 mg daily). In a phase 1 trial, of 15 DTC patients, $8(53 \%)$ had a confirmed partial response, 1 of whom saw marked improvement of a boneinfiltrating lesion; 6 (40\%), stable disease. The most common adverse events were diarrhea, hypertension, and palmarplantar erythrodysesthesia [50].

3.4. Other Targeted Therapies. Other targeted therapies that have been tested in trials in DTC patients include cyclooxygenase- 2 inhibitors, angiogenesis inhibitors, histone deacetylase inhibitors, proteasome inhibitors, and agents to restore RAI uptake. The mechanism and outcome of these agents are summarized in Table 2.

Celecoxib (400 mg twice daily). Patients were treated for 12 months in this phase 2 trial. Of 32 patients, 23 experienced progressive disease or stopped treatment because of drug toxicity, thus attaining the intent-to-treat study endpoint for 
TABLe 2: Trials of other targeted therapies in patients with metastatic differentiated thyroid cancer.

\begin{tabular}{|c|c|c|c|c|c|c|c|}
\hline Agent & $\begin{array}{l}\text { Celecoxib } \\
\quad[17]\end{array}$ & $\begin{array}{c}\text { Vorinostat } \\
{[18]}\end{array}$ & $\begin{array}{c}\text { Thalidomide } \\
\text { [19] }\end{array}$ & $\begin{array}{c}\text { Lenalidomide } \\
{[20]}\end{array}$ & $\begin{array}{c}\text { Rosiglitazone } \\
{[21]}\end{array}$ & $\begin{array}{c}\text { Bexarotene } \\
{[22]}\end{array}$ & $\begin{array}{l}\text { Selumetinib } \\
{[23]}\end{array}$ \\
\hline Mechanism & COX-2 inhibitor & $\begin{array}{c}\text { Histone } \\
\text { deacetylase } \\
\text { inhibitor }\end{array}$ & Antiangiogenesis & Antiangiogenesis & $\begin{array}{l}\text { RAIU } \\
\text { restoration }\end{array}$ & $\begin{array}{l}\text { RAIU } \\
\text { restoration }\end{array}$ & $\begin{array}{l}\text { RAIU } \\
\text { restoration }\end{array}$ \\
\hline$N$ enrolled & 32 & 19 & 36 & 25 & 25 & 11 & 12 \\
\hline $\begin{array}{l}\text { Histology } \\
\text { (DTC), } n\end{array}$ & 32 & 16 & 28 & 25 & 25 & 11 & 12 \\
\hline Outcome & RECIST & RECIST & Tumor volume & Tumor volume & Tumor volume & Tumor volume & Tumor volume \\
\hline $\mathrm{CR} \%$ & 0 & 0 & 0 & 0 & 0 & 0 & 0 \\
\hline PR\% & 3 & 0 & 18 & 22 & 20 & 0 & 42 \\
\hline SD\% & 3 & 56 & 32 & 44 & 12 & 18 & 17 \\
\hline
\end{tabular}

RAIU: radioactive iodine uptake; COX-2: cyclooxygenase-2; DTC: differentiated thyroid cancer; RECIST: response evaluation criteria in solid Tumors; CR: complete response; PR: partial response; SD: stable disease.

celecoxib failure. One patient had a partial response, and another completed 12 months of therapy progression-free. The patient with a partial response was still on celecoxib, along with 7 other patients, when the study was terminated. Adverse drug effects included infection, pain, hypertension, fainting, hypocalcemia, lymphopenia, and anemia [17].

Vorinostat (200 mg twice daily). In a phase 2 study, 16 DTC patients were treated for 2 weeks, followed by 1 week off therapy. No patient achieved a partial or complete response [18].

Thalidomide (started at $200 \mathrm{mg}$, increased over 6 weeks to $800 \mathrm{mg}$ or to the maximum tolerated dose). 28 patients were enrolled who had had various histologic types of thyroid cancer: $18 \%$ had a partial response; $32 \%$, stable disease. Outcomes were measured in terms of tumor volume, rather than with RECIST. A partial response was defined as a tumor volume reduction $>50 \%$. Most patients required dose modifications because of drug toxicity [19]. The same group of researchers who conducted the thalidomide trial reported results of a phase 2 clinical trial of lenalidomide $(25 \mathrm{mg}$ daily), with an interim analysis suggesting similar response rates to thalidomide but with less hematologic toxicity. In that subsequent report, $22 \%$ of the patients had a partial response; 44\%, stable disease [20].

Rosiglitazone (4 $\mathrm{mg}$ daily for 1 week, then $8 \mathrm{mg}$ daily for 7 weeks). In an open-label, phase 2 trial of oral rosiglitazone treatment, 20 DTC patients were enrolled: after treatment, 5 had positive RAI scan results; 5 , a partial response (decreased thyroglobulin or positive RAI uptake); 3, stable disease (no change in thyroglobulin and RAI uptake status); and 12, disease progression (increased thyroglobulin levels). By RECIST criteria, no patient, after 3 months, had a complete or partial response to rosiglitazone; on long-term followup, the trial did not result in any clinically significant response [21].
Bexarotene ( $300 \mathrm{mg}$ daily). In this phase 2 trial, 11 patients received the drug for 6 weeks: 8 had partial restoration of iodine avidity, but there was not much tumor reduction [51]. At 6 months after RAI therapy, 6 patients had progressive disease (defined as an increase $>10 \%$ in serum thyroglobulin levels and/or an increase $>25 \%$ in tumor dimensions); 2 , stable disease. Bexarotene failed to restore susceptibility to RAI therapy [22].

Selumetinib (75 $\mathrm{mg}$ twice daily). In this phase 2 trial, 20 patients received 4 weeks of selumetinib increased iodine uptake in 12 of the 20 patients ( 4 of 8 BRAF MUT; 8 of the 12 other patients). The 8 of those 12 cases achieving sufficient iodine avidity to warrant RAI therapy included all 5 patients known to be NRAS MUT and 1 BRAF MUT patient. Of the 7 pts who have received RAI, 5 had partial responses and 2 patients achieved stable disease [23].

3.5. Patient Recruitment in Clinical Trials. Referral for participation in clinical trials should be pondered for patients with progressive or symptomatic metastatic disease [52]. Various research groups in the United States and Europe are recruiting patients with DTC with radioiodine-negative progressive disease. The list of ongoing trials is summarized in Table 3.

\section{Conclusion}

With the increasing overall incidence of thyroid carcinoma worldwide, the burden of advanced and metastatic disease will rise significantly in the next few decades. Together with new discoveries of the biologic pathways of thyroid cancer, therapies specifically targeting these molecules are being formulated and presented for clinical trials. Despite the plethora of clinical trials in thyroid cancer, treatment choices for those with advanced DTC have remained extremely limited and compromised with limiting toxicities. The introduction of new treatment modalities has generated great excitement in 
TABLE 3: Ongoing comparative drugs trials in thyroid cancer.

\begin{tabular}{|c|c|c|c|c|c|}
\hline Type of thyroid cancer & Intervention & Comparison & Expected end & Current status & Identifier \\
\hline DTC* & Sorafenib & Placebo & Dec 2013 & Ongoing, not recruiting & NCT00984282 \\
\hline DTC & Lenvatinib & Placebo & Jul 2013 & Active, not recruiting & NCT01321554 \\
\hline DTC & Cediranib & Cediranib and lenalidomide & Sep 2013 & Recruiting & NCT01208051 \\
\hline DTC, MTC $^{* *}$ & Everolimus pasireotide & Everolimus or pasireotide & Jun 2014 & Recruiting & NCT01270321 \\
\hline
\end{tabular}

${ }^{*}$ DTC: differentiated thyroid cancer.

** MTC: medullary thyroid cancer.

both researchers and clinicians. However, it has to be emphasized that except the recently published Vandetanib trial [48] none of the currently available publications compared the new drug outcomes with another drug; another established therapy, placebo or no treatment control group. Currently based on phase 2 trial results, tyrosine kinase agents have shown encouraging outcomes compared to other available agents and may confer a role in the treatment of progressing and symptomatic metastatic disease. These agents should only be initiated in patients with progressive disease or significant tumor burden in the presence of RECIST targets [53].

The latest revision of RECIST (version 1.1) has incorporated some modification into the initial guidelines [53] such as reduction of number of target lesions, assessment of pathologic lymph nodes, clarification of disease progression, and inclusion of fluorodeoxyglucose positron emission tomography (FDG PET) scan in detection of new lesions, to further optimize the assessment of tumor burden [54]. However, this criteria, was originally established to measure patient's response to cytotoxic chemotherapy and may not sufficiently describe tumor response in genomically distinct subsets of patients treated with specific targeted therapies. New molecular targeted agents often induce growth inhibition rather than tumor regression and this will result in limited objective response rates according to RECIST. Nonetheless, currently, the existing data on molecular imaging (i.e., perfusion or diffusion characteristics, metabolism, and necrosis) in thyroid cancer is not adequately validated to integrate into response assessment criteria and hence, anatomical assessment of tumor burden remains the unsurpassed surrogate endpoint. Despite the limitations of the current RECIST criteria, it is crucial that researchers report their outcomes in a standardized fashion and not according to solely clinical determinants to allow data comparison across diverse demographics and different institutions.

Future direction towards "personalized" tumor treatment based on genotyping may demand tumor assessment tools such as newly proposed Choi criteria that ultimately include functional and molecular imaging rather than anatomical assessment of tumor size [55]. It is also fundamental to standardize recruitment policies in order to register advanced DTC patients in appropriate phase 1 or 2 trials to foster new compounds or combination therapies that eventually will lead to better management or cure in these patients.

\section{References}

[1] S. I. Sherman, "Thyroid carcinoma," The Lancet, vol. 361, no. 9356, pp. 501-511, 2003.

[2] L. Agate, L. Lorusso, and R. Elisei, "New and old knowledge on differentiated thyroid cancer epidemiology and risk factors," Journal of Endocrinological Investigation, vol. 35, no. 6, pp. 39, 2012.

[3] American Cancer Society, Cancer Facts and Figures 2012, American Cancer Society, Atlanta, Ga, USA, 2012.

[4] A. Antonelli, P. Fallahi, S. M. Ferrari et al., "Dedifferentiated thyroid cancer: a therapeutic challenge," Biomedicine and Pharmacotherapy, vol. 62, no. 8, pp. 559-563, 2008.

[5] R. M. Tuttle, "Novel therapeutic options for aggressive thyroid cancer: integrating information from the recent clinical trials into clinical practice," Clinical Thryoidology, vol. 21, pp. 3-7, 2009.

[6] M. Xing, "Recent advances in molecular biology of thyroid cancer and their clinical implications," Otolaryngologic Clinics of North America, vol. 41, no. 6, pp. 1135-1146, 2008.

[7] S. I. Sherman, L. J. Wirth, J. P. Droz et al., "Motesanib diphosphate in progressive differentiated thyroid cancer," New England Journal of Medicine, vol. 359, no. 1, pp. 31-42, 2008.

[8] E. E. W. Cohen, L. S. Rosen, E. E. Vokes et al., "Axitinib is an active treatment for all histologic subtypes of advanced thyroid cancer: results from a phase II study," Journal of Clinical Oncology, vol. 26, no. 29, pp. 4708-4713, 2008.

[9] V. Gupta-Abramson, A. B. Troxel, A. Nellore et al., "Phase II trial of sorafenib in advanced thyroid cancer," Journal of Clinical Oncology, vol. 26, no. 29, pp. 4714-4719, 2008.

[10] R. T. Kloos, M. D. Ringel, M. V. Knopp et al., "Phase II trial of sorafenib in metastatic thyroid cancer," Journal of Clinical Oncology, vol. 27, pp. 1675-1684, 2009.

[11] H. Hoftijzer, K. A. Heemstra, H. Morreau et al., "Beneficial effects of sorafenib on tumor progression, but not on radioiodine uptake, in patients with differentiated thyroid carcinoma," European Journal of Endocrinology, vol. 161, no. 6, pp. 923-931, 2009.

[12] M. Ahmed, Y. Barbachano, A. Riddell et al., "Analysis of the efficacy and toxicity of sorafenib in thyroid cancer: a phase II study in a UK based population," European Journal of Endocrinology, vol. 165, no. 2, pp. 315-322, 2011.

[13] V. Marotta, V. Ramundo, L. Camera et al., "Sorafenib in advanced iodine-refractory differentiated thyroid cancer: efficacy, safety and exploratory analysis of role of serum thyroglobulin and FDG-PET," Clinical Endocrinology. In press.

[14] L. L. Carr, D. A. Mankoff, B. H. Goulart et al., "Phase II study of daily sunitinib in FDG-PET-positive, iodine-refractory 
differentiated thyroid cancer and metastatic medullary carcinoma of the thyroid with functional imaging correlation," Clinical Cancer Research, vol. 16, no. 21, pp. 5260-5268, 2010.

[15] E. E. Cohen, B. M. Needles, K. J. Cullen et al., "Phase 2 study of sunitinib in refractory thyroid cancer," Journal of Clinical Oncology, vol. 26, no. 20, article 6025, 2008.

[16] K. C. Bible, V. J. Suman, J. R. Molina et al., "Efficacy of pazopanib in progressive, radioiodine-refractory, metastatic differentiated thyroid cancers: results of a phase 2 consortium study," The Lancet Oncology, vol. 11, no. 10, pp. 962-972, 2010.

[17] E. Mrozek, R. T. Kloos, M. D. Ringel et al., "Phase II study of celecoxib in metastatic differentiated thyroid carcinoma," Journal of Clinical Endocrinology and Metabolism, vol. 91, no. 6, pp. 2201-2204, 2006.

[18] J. A. Woyach, R. T. Kloos, M. D. Ringel et al., "Lack of therapeutic effect of the histone deacetylase inhibitor vorinostat in patients with metastatic radioiodine-refractory thyroid carcinoma," Journal of Clinical Endocrinology and Metabolism, vol. 94, no. 1, pp. 164-170, 2009.

[19] K. B. Ain, C. Lee, and K. D. Williams, "Phase II trial of thalidomide for therapy of radioiodine-unresponsive and rapidly progressive thyroid carcinomas," Thyroid, vol. 17, no. 7, pp. 663-670, 2007.

[20] K. B. Ain, C. Lee, K. Holbrook, J. Dziba, and K. Williams, "Phase II study of lenalidomide in distantly metastatic, rapidly progressive, and radioiodine-unresponsive thyroid carcinomas: preliminary results," Journal of Clinical Oncology, 6027, 2008.

[21] E. Kebebew, S. Lindsay, O. H. Clark, K. A. Woeber, R. Hawkins, and F. S. Greenspan, "Results of rosiglitazone therapy in patients with thyroglobulin-positive and radioiodine-negative advanced differentiated thyroid cancer," Thyroid, vol. 19, no. 9, pp. 953-956, 2009.

[22] Y. Y. Liu, M. P. Stokkel, H. A. Morreau, A. M. Pereira, J. A. Romijn, and J. W. A. Smit, "Radioiodine therapy after pretreatment with bexarotene for metastases of differentiated thyroid carcinoma," Clinical Endocrinology, vol. 68, no. 4, pp. 605-609, 2008.

[23] L. Ho, R. K. Grewal, R. Leboeuf et al., "Reacquisition of RAI uptake in RAI-refractory, metastatic thyroid cancers by pretreatment with the selective MEK inhibitor, selumetinib," Journal of Clinical Oncology, vol. 30, article 5509, 2012.

[24] V. Trovisco, P. Soares, and M. Sobrinho-Simões, "B-RAF mutations in the etiopathogenesis, diagnosis, and prognosis of thyroid carcinomas," Human Pathology, vol. 37, no. 7, pp. 781-786, 2006.

[25] R. Ciampi and Y. E. Nikiforov, "Alterations of the BRAF gene in thyroid tumors," Endocrine Pathology, vol. 16, no. 3, pp. 163-172, 2005.

[26] M. Xing, "BRAF mutation in thyroid cancer," EndocrineRelated Cancer, vol. 12, no. 2, pp. 245-262, 2005.

[27] O. Gimm, "Thyroid cancer," Cancer Letters, vol. 163, no. 2, pp. 143-156, 2001.

[28] J. Rosai, "Handling of thyroid follicular patterned lesions," Endocrine Pathology, vol. 16, no. 4, pp. 279-284, 2005.

[29] R. M. Quiros, H. G. Ding, P. Gattuso, R. A. Prinz, and X. Xu, "Evidence that one subset of anaplastic thyroid carcinomas are derived from papillary carcinomas due to BRAF and p53 mutations," Cancer, vol. 103, no. 11, pp. 2261-2268, 2005.

[30] G. Tallini and S. L. Asa, "RET oncogene activation in papillary thyroid carcinoma," Advances in Anatomic Pathology, vol. 8, no. 6, pp. 345-354, 2001.
[31] E. T. Kimura, M. N. Nikiforova, Z. Zhu, J. A. Knauf, Y. E. Nikiforov, and J. A. Fagin, "High prevalence of BRAF mutations in thyroid cancer: genetic evidence for constitutive activation of the RET/PTC-RAS-BRAF signaling pathway in papillary thyroid carcinoma," Cancer Research, vol. 63, no. 7, pp. 14541457, 2003.

[32] V. Marotta, A. Guerra, M. R. Sapio, and M. Vitale, "RET/PTC rearrangement in benign and malignant thyroid diseases: a clinical standpoint," European Journal of Endocrinology, vol. 165, no. 4, pp. 499-507, 2011.

[33] V. Marotta, M. R. Sapio, A. Guerra, and M. Vitale, "BRAF mutation in cytology samples as a diagnostic tool for papillary thyroid carcinoma," Expert Opinion on Medical Diagnostics, vol. 5, no. 4, pp. 277-290, 2011.

[34] H. M. Rabes, E. P. Demidchik, J. D. Sidorow et al., "Pattern of radiation-induced RET and NTRK1 rearrangements in 191 post-Chernobyl papillary thyroid carcinomas: biological, phenotypic, and clinical implications," Clinical Cancer Research, vol. 6, no. 3, pp. 1093-1103, 2000.

[35] M. N. Nikiforova, R. A. Lynch, P. W. Biddinger et al., "RAS point mutations and PAX8-PPAR $\gamma$ rearrangement in thyroid tumors: evidence for distinct molecular pathways in thyroid follicular carcinoma," Journal of Clinical Endocrinology and Metabolism, vol. 88, no. 5, pp. 2318-2326, 2003.

[36] A. R. Marques, C. Espadinha, A. L. Catarino et al., "Expression of PAX8-PPAR $\gamma 1$ rearrangements in both follicular thyroid carcinomas and adenomas," Journal of Clinical Endocrinology and Metabolism, vol. 87, no. 8, pp. 3947-3952, 2002.

[37] O. Gimm, A. Perren, L. P. Weng et al., "Differential nuclear and cytoplasmic expression of PTEN in normal thyroid tissue, and benign and malignant epithelial thyroid tumors," American Journal of Pathology, vol. 156, no. 5, pp. 1693-1700, 2000.

[38] M. D. Ringel, N. Hayre, J. Saito et al., "Overexpression and overactivation of Akt in thyroid carcinoma," Cancer Research, vol. 61, no. 16, pp. 6105-6111, 2001.

[39] P. Bruni, A. Boccia, G. Baldassarre et al., "PTEN expression is reduced in a subset of sporadic thyroid carcinomas: evidence that PTEN-growth suppressing activity in thyroid cancer cells is mediated by p27(kip1)," Oncogene, vol. 19, no. 28, pp. 31463155, 2000.

[40] Z. Liu, P. Hou, M. Ji et al., "Highly prevalent genetic alterations in receptor tyrosine kinases and phosphatidylinositol 3kinase/Akt and mitogen-activated protein kinase pathways in anaplastic and follicular thyroid cancers," Journal of Clinical Endocrinology and Metabolism, vol. 93, no. 8, pp. 3106-3116, 2008.

[41] V. Vasko, M. Ferrand, J. Di Cristofaro, P. Carayon, J. F. Henry, and C. de Micco, "Specific pattern of RAS oncogene mutations in follicular thyroid tumors," Journal of Clinical Endocrinology and Metabolism, vol. 88, no. 6, pp. 2745-2752, 2003.

[42] M. Fassnacht, M. C. Kreissl, D. Weismann, and B. Allolio, "New targets and therapeutic approaches for endocrine malignancies," Pharmacology and Therapeutics, vol. 123, no. 1, pp. 117-141, 2009.

[43] S. I. Sherman, "Targeted therapy of thyroid cancer," Biochemical Pharmacology, vol. 80, no. 5, pp. 592-601, 2010.

[44] M. Klein, J. M. Vignaud, V. Hennequin et al., "Increased expression of the vascular endothelial growth factor is a pejorative prognosis marker in papillary thyroid carcinoma," Journal of Clinical Endocrinology and Metabolism, vol. 86, no. 2, pp. 656-658, 2001.

[45] E. Y. Soh, M. S. Eigelberger, K. J. Kim et al., "Neutralizing vascular endothelial growth factor activity inhibits thyroid 
cancer growth in vivo," Surgery, vol. 128, no. 6, pp. 1059-1066, 2000.

[46] J. Capdevila, L. Iglesias, I. Halperin et al., "Sorafenib in metastatic thyroid cancer," Endocrine-Related Cancers, vol. 19, no. 2, pp. 209-216, 2012.

[47] S. I. Sherman, B. Jarzab, M. E. Cabanillas et al., "A phase II trial of the multi-targeted kinase inhibitor, Lenvatinib (E7080), in advanced radioiodine-refractory differentiated thyroid cancer (DTC)," Journal of Clinical Oncology, vol. 29, no. 15, article 5503, 2011.

[48] S. Leboulleux, L. Bastholt, T. Krause et al., "Vandetanib in locally advanced or metastatic differentiated thyroid cancer: a randomised, double-blind, phase 2 trial," The Lancet Oncology, vol. 13, no. 9, pp. 897-905, 2012.

[49] D. S. Hong, M. E. Cabanillas, J. Wheler et al., "Inhibition of the ras/raf/MEK/ERK and RET kinase pathways with the combination of the multikinase inhibitor sorafenib and the farnesyltransferase inhibitor tipifarnib in medullary and differentiated thyroid malignancies," Journal of Clinical Endocrinology and Metabolism, vol. 96, no. 4, pp. 997-1005, 2011.

[50] M. E. Cabanillas, M. S. Brose, D. A. Ramies et al., "Anti-tumor activity observed in a cohort of patients with differentiated thyroid cancer in a phase 1 study of cabozantinib (XL184)," Journal of Clinical Oncology, p. 5547, 2012.

[51] Y. Y. Liu, M. P. Stokkel, A. M. Pereira et al., "Bexarotene increases uptake of radioiodide in metastases of differentiated thyroid carcinoma," European Journal of Endocrinology, vol. 154, no. 4, pp. 525-531, 2006.

[52] D. S. Cooper, G. M. Doherty, B. R. Haugen et al., "Revised American thyroid association management guidelines for patients with thyroid nodules and differentiated thyroid cancer," Thyroid, vol. 19, no. 11, pp. 1167-1214, 2009.

[53] P. Therasse, S. G. Arbuck, E. A. Eisenhauer et al., "New guidelines to evaluate the response to treatment in solid tumors (RECIST guidelines)," Journal of the National Cancer Institute, vol. 92, pp. 205-216, 2000.

[54] E. A. Eisenhauer, P. Therasse, J. Bogaerts et al., "New response evaluation criteria in solid tumours: revised RECIST guideline (version 1.1)," European Journal of Cancer, vol. 45, no. 2, pp. 228-247, 2009.

[55] M. Mizuki Nishino, J. P. Jagannathan, and K. M. Krajewski, "Personalized tumor response assessment in the era of molecular medicine: cancer-specific and therapy-specific response criteria to complement pitfalls of RECIST," American Journal of Roentgenology, vol. 198, no. 4, pp. 737-745, 2012. 


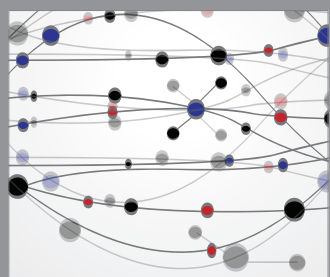

The Scientific World Journal
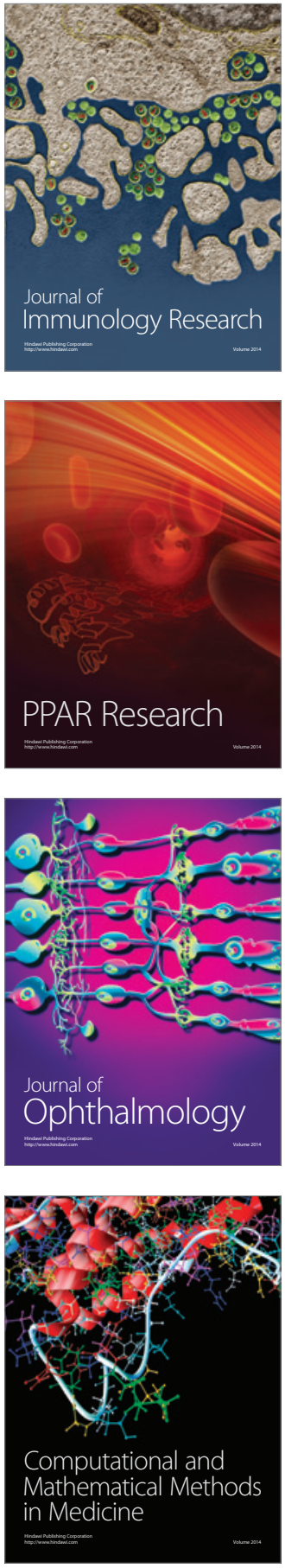

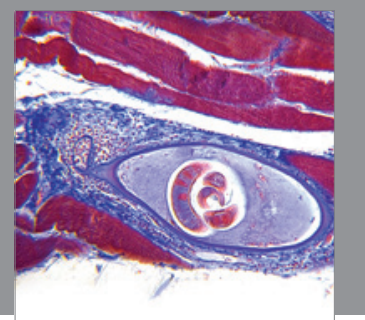

Gastroenterology

Research and Practice
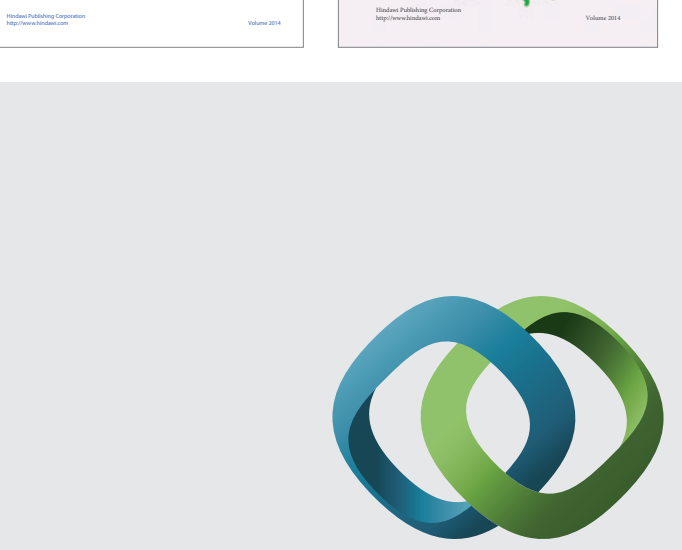

\section{Hindawi}

Submit your manuscripts at

http://www.hindawi.com
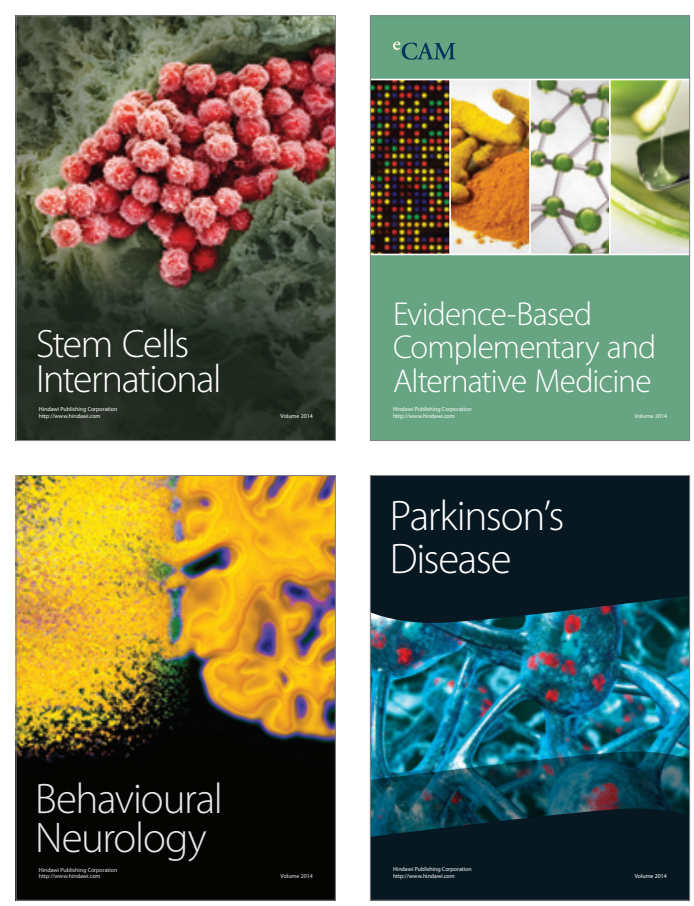

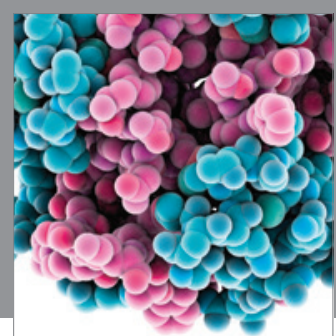

Journal of
Diabetes Research

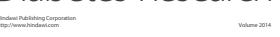

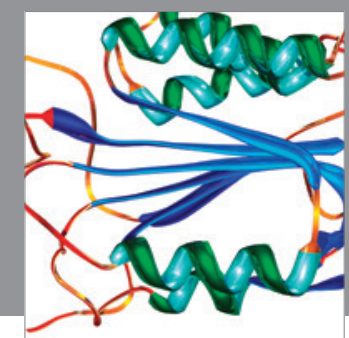

Disease Markers
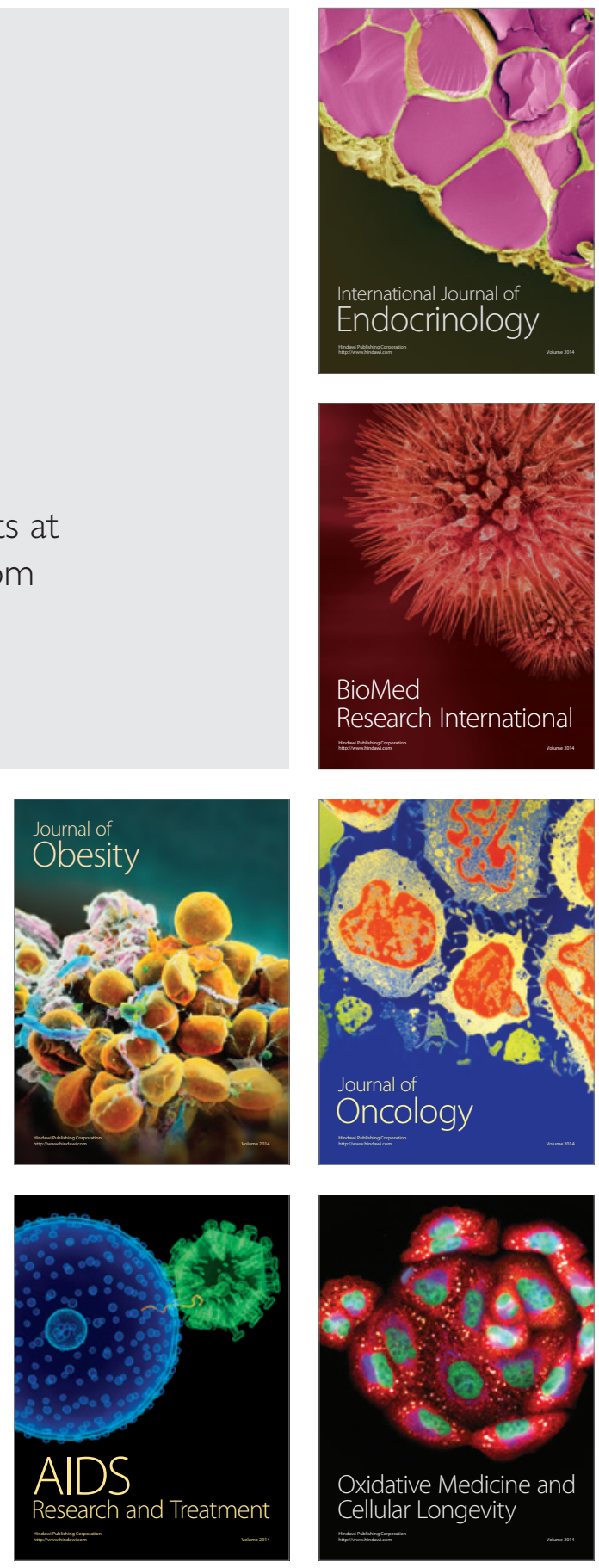